\title{
Capacidades críticas y acríticas de los estudiantes de noveno grado de instituciones de educación media de Cúcuta, Pamplona y Villa del Rosario frente a los contenidos audiovisuales de Facebook y YouTube. Caso: Colegio Departamental Integrado General Santander*
}

Hugo Alexander Vega Riaño', D orcid.org/0000-0002-9792-4685 Marcela Bibiana Villamizar Castro ${ }^{2}$ Universidad de Pamplona, Colombia

\section{Critical and non critical capabilities of ninth grade students of high school institutions in Cucuta, Pamplona and Villa del Rosario, compared with audiovisual content of Facebook and YouTube. Case: Integrated Departmental General Santander School}

Palabras clave:

Influencia e impacto de las nuevas tecnologías,

Interacción humana,

Procesos de socialización e

interacción,

Capacidades críticas o acríticas.

Keywords:

Influence and impact of

new technologies,

Human interaction,

Processes of socialization and

interaction,

Critical and non critical

capabilities.

\begin{abstract}
Resumen
Al considerar la influencia e impacto de las nuevas tecnologías de la información y la comunicación (TIC) en los procesos de interacción humana, se conciben estas herramientas como manifestaciones que median la forma de pensar y actuar de las personas que a diario las utilizan. Estas plataformas de comunicación deben tener destacada atención por cuanto se erigen como medios determinantes en procesos de socialización e interacción en los que se trascienden fronteras de tipo personal y geográfico. A través de sus contenidos, dichas tecnologías han modificado los modos de ser, pensar, sentir e interactuar de los seres humanos, especialmente de aquellos de más temprana generación, y que en este caso refiere a la determinación de las capacidades críticas o acríticas de los jóvenes de noveno grado de educación media de Cúcuta, Pamplona y Villa del Rosario (Colombia) en presencia de redes y sitios web como Facebook y YouTube.
\end{abstract}

\begin{abstract}
When you consider the influence and impact of new technologies in the information and communication (TIC) processes of human relations, these tools are always understood as expressions that mediate the way of thinking and acting of the daily users. These communication platforms must be given an outstanding attention for they appear as determinant factors in the process of socialization and interaction, in which they transcend personal and geographic boundaries. Through their contents, such technologies have changed the ways of behavior, thought, emotional well- being as well as interaction in human beings, particularly those of earlier generations, and in this case it refers to the determination of their critical and non critical capabilities of ninth grade high school youths in Cucuta, Pamplona and Villa del Rosario (Colombia) with the presence of nets and websites like Facebook and YouTube.
\end{abstract}

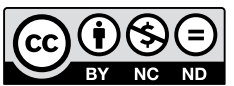

Referencia de este artículo (APA): Vega, H. \& Villamizar, M. (2016). Capacidades críticas y acríticas de los estudiantes de noveno grado de instituciones de educación media de Cúcuta, Pamplona y Villa del Rosario frente a los contenidos audiovisuales de Facebook y YouTube. Caso: Colegio Departamental Integrado General Santander. Revista Educación y Humanismo, 18(31), $274-301$. http://dx.doi.org/10.17081/eduhum.18.31.1379

\footnotetext{
Artículo resultado de la investigación: Inclusión, educación y cultura. Capacidades críticas y acríticas de los estudiantes de la ciudad de Cúcuta. Universidad de Pamplona, Norte de Santander.

1. Universidad de Pamplona, Colombia. Email: havega@gmail.com

2. Universidad de Pamplona, Colombia. Email: marcelavillamizar@hotmail.com
} 


\section{Introducción}

"En la cultura de la televisión, los ordenadores y las telecomunicaciones una nueva visión del aprendizaje está emergiendo" (Ballesta, 2002, p.239). Reforzando lo afirmado por el autor, si bien es cierto que la escuela (tomada en cuanto el espacio formal donde se desarrollan procesos de enseñanza-aprendizaje) ha sido validada desde hace siglos como el lugar donde el conocimiento se forja, se imparte, comparte, se edifica y solidifica, en la actualidad, con la aparición de los medios masivos de comunicación y de las llamadas tecnologías de información y comunicación (TIC), dicho conocimiento ha trascendido las barreras de las estructuras formales, otrora consideradas como sagradas, causando una deslocalización que rompe incluso con el concepto de lo físico en tanto que ha desbordado los libros, contemplados tiempo atrás como los dispositivos técnicos privilegiados para contener información (Cárdenas, 2008).

De la misma manera, los tiempos establecidos para lograr el aprendizaje con rutas y delimitaciones han sufrido un proceso que bien se podría llamar des-temporalización, pues cada quien aprende conforme sus propios ritmos y rutas, sin otra limitación que la que marca su proceso personal.

Es decir, con la llegada de la globalización, la aparición de los medios y la incursión de las tecnologías en nuestras sociedades, la población sujeto-objeto de la educación ya no solo aprende, ya no llega a la fuente del conocimiento tan solo para construirlo en un claustro sagrado a través de un libro y con un docente como figura experta en el mundo de las ciencias y disciplinas conceptuales (Blázquez, 2001). En otras palabras, los medios de comunicación y las tecnologías de la información se han constituido, y cada vez de manera más creciente, en lugares caracterizados por la virtualidad, la rapidez, la instantaneidad, la multimedia, la interactividad y la interconexión (García \& Álvarez, 2001), entre otras, donde también se aprende o se desaprende, se experimenta y se construyen saberes, algunos útiles y otros no tanto, pero que en muchos casos llegan a ser más atractivos y más influyentes en los niños y jóvenes de la educación actual que los de la propia escuela.

Tal situación recompone hoy en día no solo los lugares, las figuras sociales y los dispositivos para facilitar el aprendizaje, invitando a la educación formal a repensarse y vincular a sus procesos las bondades, ventajas y utilidades que pueden contener las TIC y los medios masivos (mass media) en la construcción del conocimiento, y a asumir una postura crítica y reflexiva sobre los mismos. Esta idea va en contravía de algunas posturas que por mucho tiempo han imperado entre quienes tienen la misión de administrar la educación, pues no ha sido raro encontrar opiniones de directivos y docentes que aún en la actualidad satanizan y, en el mejor de los casos, relegan a las TIC y a los medios masivos a espacios físicos en donde se resguardan sigilosamente equipos bajo llave que controla un "encargado", única persona autorizada para 
manipularlos. Bajo esta dinámica, muchas instituciones funcionan perdiendo la oportunidad de sacar el máximo de ventaja de una tecnología que en lugar de rival puede resultar siendo la aliada perfecta cuando de enseñar y aprender se trata.

Lo anteriormente mencionado marca el punto de encuentro entre dos disciplinas que podrían pensarse como el binomio perfecto en la construcción del conocimiento y los saberes formales, e incluso de los no formales: la educación y la comunicación, o como también lo llamaría Paulo Freire o Mario Kaplún, la educomunicación, término que se refiere a que el hecho educativo es, en esencia, un hecho comunicativo (Aparici, 2011). Este vocablo, aceptado por la Unesco en 1979, plantea que los procesos de comunicación son componentes pedagógicos del aprendizaje y, más aún: que la comunicación puede estar al servicio de la educación en cuanto los medios y las tecnologías son herramientas para generar, facilitar, transmitir y edificar saberes (Ministerio de Educación Nacional, 2013). En este caso, se estaría hablando de la primera vertiente de la educomunicación, es decir, de la comunicación para la educación.

Así mismo, y como segunda vertiente de este binomio, la educación también puede estar al servicio de la comunicación, en cuanto sirve como alfabetizadora para aprender a leer, interpretar y analizar críticamente los códigos, mensajes y contenidos que circulan a través de las tecnologías y los mass media.
Y es aquí, en este punto, donde se centra la atención del presente documento. La educación para la comunicación plantea un reto a la escuela en sus diferentes niveles (básica, media y superior) debido a que de las dos vertientes de la educomunicación es la que quizás menos se ha implementado. Esto significa que existe una necesidad latente de brindar a los jóvenes acompañamiento, adiestramiento, orientación y argumentos para que la sociedad de la información en la que se encuentran sumergidos no los llene de vastos datos, de gran cantidad de información y de una serie de productos mediáticos que en lugar de contribuirles a su formación como seres humanos integrales, terminan desorientándolos y manipulando sus pensamientos, actuaciones y conductas, al punto de convertirlos en ciudadanos pasivos.

Intentar el abordaje de los medios de comunicación y las tecnologías de la información desde la escuela busca reconocer el poder que estos tienen para llegar e influir en los niños y jóvenes en cuanto le presentan en tiempo real y con imágenes, sonidos y formatos llamativos de ideas, mensajes, valores, estereotipos, antivalores y tendencias que han sido creadas por unos pocos para ser consumidas por muchos.

Las instituciones educativas deben abrir espacios de diálogo y reflexión desde una postura crítica para llevar a los estudiantes a interrogarse sobre los productos mediáticos que se encuentran a su alcance, propiciándoles herramientas $\mathrm{y}$ argumentos para que hagan las lecturas ade- 
cuadas sobre ellos sin caer en el encanto de su dominación y manipulación. La escuela debe favorecer que los alumnos entiendan y relacionen el mundo comunicativo que experimentan fuera de las aulas, en su cotidianidad, con el mundo de conocimientos y saberes que se aprenden dentro de la institución.

Realizar esto requiere de un proceso con varias etapas: primero, lograr que cale el discurso educomunicativo y de recepción crítica de medios en las instituciones educativas; segundo, capacitar a administrativos y docentes en el manejo e interpretación adecuada de los medios y sus contenidos, es decir, alfabetizar primero a quienes deberán después llevar el proceso a los escolares. Como tercera etapa se encontraría la de generar los espacios para que los estudiantes reciban la formación necesaria y empiecen a comprender que, como dice Masterman (1993):

Los medios tienen una importancia ideológica tan enorme que se hace necesaria una educación audiovisual con urgencia en las escuelas porque quienes controlan y trabajan en los medios no solo tienen poder para fijar prioridades, ofrecer explicaciones y construir sus propias versiones de los acontecimientos, sino, que además tienen $[(\ldots)$ una] capacidad mucho más importante: [...] proyectar estos acontecimientos como naturales y auténticos, sencillamente como una parte de la manera de ser de las cosas. Es el poder de mitificar, de amplificar, de convertir una situación en un gran acontecimiento o a una persona de a pie en todo un héroe. El hecho de rellenar un dato o simplemente omitir[lo] se convierte en una manera de trascender y optar por una ideología, tomar partido, destacar o subrayar una opción determinada (p.20).

Lograr generar este proceso consolidará la idea de una ciudadanía (compuesta por niños y jóvenes) activa, crítica y veedora de los contenidos ante los cuales decidirá su postura y formará sus propios veredictos, desechando lo que no le resulte útil y acogiendo lo que mejor sirva para su formación y desarrollo humano.

\section{La cultura comunicativa a partir del desa-} rrollo tecnológico

El mundo actual se encuentra caracterizado por el auge y el desarrollo tecnológico, el cual ha impactado todas las dimensiones y esferas de los seres humanos hasta tal punto que se crean y socializan valores que legitiman este tipo de sociedad (Murciano, 2006). Se puede afirmar que el desarrollo tecnológico ha permitido configurar la actual sociedad y las estructuras culturales, económicas, políticas y sociales que definen cada uno de los contextos donde se encuentra inmersa la humanidad.

De este modo, identificar el impacto tecnológico desde un punto de vista global resultaría osado, pues son múltiples y muy variados los escenarios donde la tecnología ha tenido influencia: 
Los efectos y el alcance, no solo se sitúan en el terreno de la información y comunicación, sino que lo sobrepasan para llegar a provocar y proponer cambios en la estructura social, económica, laboral, jurídica y política. Y ello es debido a que no solo se centran en la captación de la información, sino también, y es lo verdaderamente significativo, en las posibilidades que tienen para manipularla, almacenarla y distribuirla (Cabero, 1996).

Sin embargo, es posible estudiar dicho fenómeno atendiendo a los campos y niveles donde el desarrollo tecnológico ha tenido algún efecto, por ejemplo, el campo de la cultura comunicativa.

Algunos términos permiten definir de forma más precisa la actual dinámica del mundo. Dentro de estos se encuentran la denominada sociedad del conocimiento, la cual prioriza las redes, la conectividad, el manejo de la información y la construcción del conocimiento colectivo apoyado en un conjunto de factores como las nuevas tecnologías. En otros términos, la sociedad del conocimiento ha tenido un impacto profundo en el ámbito social, económico, político y cultural desde su nacimiento en la década los noventa, cuando se reemplazó con esta denominación la sociedad de la información (Rodríguez, 2009; Druetta, 2002).

Tubella y Requena (2005) exponen cómo la sociedad del conocimiento se concreta en una estructura de redes impactando la morfología de las actuales sociedades; a su vez, Castells (2006) destaca que la figura de la sociedad del conocimiento representa el uso del saber y el conocimiento como parámetros que estructuran y condicionan la sociedad, y Marqués (2001) señala que este tipo de sociedad se origina en los cambios tecnológicos y económicos, en donde el conocimiento representa el principal factor de éxito.

Cada uno de los autores expuestos pone de manifiesto que el desarrollo tecnológico ha sido una pieza clave en la construcción y reproducción de la actual sociedad del conocimiento. Esta es una sociedad centrada en el conocimiento, toda vez que dicho elemento, ya sea formal o informal, es producido, socializado, interiorizado, reelaborado y puesto en práctica para impulsar nuevas transformaciones en el campo político, social, cultural y económico.

En efecto, si bien las apreciaciones de los autores son heterogéneas, hay un aspecto en el que convergen todos: hablar de sociedad del conocimiento implica hacer referencia ineludible al desarrollo y uso de las tecnologías (Druetta, 2002; Tubella \& Requena, 2005), pues constituyen medios para la transferencia de la información y la configuración de otros escenarios de comunicación.

No se trata de un asunto nuevo. En los últimos 30 años ha tenido un auge exponencial la literatura sobre el desarrollo tecnológico y su impacto en la vida de los hombres y mujeres a 
nivel global. Por lo anterior, se habla de una revolución que se enfoca en las tecnologías de la información y la comunicación, el uso y expansión de la Internet, los adelantos en los campos de las ciencias exactas como la ingeniería, la genética, la medicina, entre otras (Castells, 2002). Todo esto se encuentra estrechamente relacionado con el sistema económico, la dinámica de los mercados y los objetivos de nivel mercantil creados por el neoliberalismo. Productividad y competencia son dos conceptos que se relacionan con el desarrollo tecnológico y esto ha servido de base para fundamentar y legitimar el actual progreso en materia tecnológica.

Sin embargo, una visión crítica sobre este fenómeno permite denunciar la forma en que el desarrollo tecnológico se pone a disposición de las dinámicas y formas de poder predominantes, esto es, el poder económico. Sin duda se observa que el desarrollo tecnológico ha servido en gran medida para legitimar y sustentar un sistema económico que ha traído consecuencias nefastas y profundas al mundo. Si bien no es objetivo de este ensayo ahondar en estos aspectos, resulta importante señalar esta perspectiva, pues las intencionalidades que se tejen detrás del desarrollo tecnológico pueden también extenderse a la misma comunicación que este proceso influencia.

Entonces, la comunicación juega un rol fundamental en el desarrollo tecnológico mientras el desarrollo de nuevas tecnologías impacta el ámbito de la comunicación. De allí que MartínBarbero (1992) describa que "sin centro [...] la sociedad se da como modelo la comunicación: red de conexión entre todos sus espacios y funciones, autorregulación que es equilibrio y circulación, retroacción constante, y transparencia, esto es, correspondencia entre todos los saberes en el código/idioma de la información" (p.4). En otros términos, los mismos sujetos y grupos sociales reconfiguran los modelos de comunicación utilizados en cuanto a funciones, criterios, relaciones, circulación, recepción, etcétera. Por tanto, el desarrollo tecnológico por sí mismo no genera las estructuras del modelo comunicativo, pues esto les corresponde a los hombres y mujeres que se encuentran inmersos en la comunicación.

Luego es necesario distinguir que el binomio comunicación-desarrollo tecnológico no puede ser explicado de una forma simplista y lineal que reduzca la comunicación a los mismos medios o instrumentos tecnológicos (Martín-Barbero, 1992). Por el contrario, las relaciones que subyacen entre estas dos esferas deben ser explicadas desde las mismas prácticas, significados e intencionalidades de los sujetos. Esto se traduce en estudios de la comunicación que van más allá de los medios, los canales y los mismos mensajes, para centrarse en los sujetos que crean y reconstruyen el acto comunicativo.

Martín-Barbero (1984) menciona que la comunicación hasta ahora se ha venido estudiando de manera inadecuada, o al menos ha debido "perder el objeto para ganar el proceso" (p.24). El objeto se refiere a los mismos puntos de in- 
terés que durante décadas se han venido insertando en los estudios de la comunicación como los medios o canales, y que poco contribuyen a comprender e interpretar la comunicación desde los sujetos, es decir, desde el punto central de producción y socialización de la información.

Para el autor en mención, los desarrollos teórico-conceptuales en torno a la comunicación deben, en estos tiempos, abordarse desde la cultura alejándose de la idea de la comunicación como una "problemática de los medios, los canales y los mensajes", es decir, de la óptica instrumentalista que ha buscado el control de las sociedades a través de la comunicación. La cultura de los sujetos se comprende como saberes, prácticas, significados, creencias y emociones que se construyen y socializan, y que de una manera u otra dan sentido a la comunicación. La finalidad o la intencionalidad de los sujetos en la comunicación es clave para comprender cómo se ha impactado este proceso a partir del desarrollo tecnológico.

Para Martín-Barbero (1984) la comunicación debe ser observada desde los mismos procesos de comunicación gestados en las comunidades, entre los grupos sociales. La manera como usan el lenguaje, los canales que utilizan, el contenido de lo que comunican, las razones por las que comunican y la cultura que enmarca toda esta dinámica de retroalimentación son de necesario análisis para el estudio y uso de la comunicación. La propuesta aquí planteada es clara: es necesario abandonar el paradigma informacional de la co- municación así como su sentido positivista para encontrar más allá de los métodos el nuevo objeto de estudio de la comunicación.

A su vez, Thompson (1998) señala que la comunicación es una actividad social en la que se producen, transmiten y recepcionan formas simbólicas, dando un significado a la realidad de los sujetos. Del mismo modo, en el proceso de comunicación, el receptor no es un sujeto pasivo e inamovible. Por el contrario, las denominadas masas tienen la capacidad de tomar posturas críticas frente a la comunicación de la que son partícipes.

En términos finalísticos, el desarrollo tecnológico no impacta de manera aislada y por casualidad la comunicación. Existe un conjunto de propósitos de naturaleza económica, por lo general, que son promovidos a partir del desarrollo tecnológico al cimentar nuevas dinámicas comunicativas entre los sujetos: mayores niveles de consumo, expansión de mercados, aumento de la producción, etcétera.

Entre tanto, otro tipo de efectos tiene el desarrollo tecnológico como el de disminuir la capacidad crítica de los sujetos, que poco o nada reflexionan sobre los contenidos a los cuales son expuestos y que ellos mismos reproducen legitimando un orden social de naturaleza económico-global. Entonces, es necesario formar a los sujetos en habilidades y competencias para la recepción de la información, capacidades para la interpretación de los productos mediáticos y para 
la ubicación de estos productos comprendiendo un momento histórico determinado (Thompson, 1998).

Por otro lado, se observa que el desarrollo de nuevas tecnologías tiene un impacto en la construcción de escenarios comunicativos alternativos. Sobre este asunto Cabero (1996) explica que el desarrollo tecnológico

[...] crea entornos tanto humanos como artificiales de comunicación no conocidos hasta la actualidad, y establecen nuevas formas de interacción de los usuarios con las máquinas donde uno y otra desempeñan roles diferentes a los clásicos de receptor y transmisor de información, y el conocimiento contextualizado se construye en la interacción que sujeto y máquina establezcan (p.2).

La anterior descripción indica que el desarrollo tecnológico promociona cada vez más diversos espacios para la comunicación. Ya sean reales o virtuales, estos escenarios de comunicación aumentan la cantidad de información a transmitir y permiten la generación de nuevos significados que impactan la forma de entender y pensar el mundo. La distancia y el tiempo se modifican, es decir, el aquí y el ahora se reconfiguran, para permitir un mayor acercamiento del hombre con la información. Sin embargo, debe ser objeto de análisis y preocupación la disminución de los escenarios que permiten la comunicación entre los hombres de forma natural y humana, la cual no puede ser reemplazada por el desarrollo tecnológico.

\section{Educomunicación: recepción crítica de} medios

En un mundo cuya sociedad se mueve en red, y que en palabras de Castells (1999) "se caracteriza por la globalización de la economía, la organización en redes, la flexibilidad e inestabilidad del trabajo, la individualización, la cultura virtual construida a través de medios de comunicación omnipresentes, interconectados y diversificados y la transformación de los cimientos materiales de la vida, el espacio y el tiempo" (pp.23-24). Se observa cómo dicha sociedad se mueve a una velocidad vertiginosa. Las nuevas tecnologías de la información diversifican diariamente sus posibilidades. Cada día surgen nuevos medios, soportes y fuentes informativas. Con ello, nuevos lenguajes y, en sí, otras formas de comunicarse se dan entre los seres humanos; algunas sencillas, otras amables, pero casi siempre confusas y complejas.

Lo anterior conlleva a plantearse incógnitas que científicos y estudiosos han tratado de responder en el papel, y que buscan, como en el caso del presente proyecto, encontrar sustento en la experiencia, en lo real y a partir de procesos de investigación. $\mathrm{Y}$ es que todas las personas, especialmente las más jóvenes, reciben multitud de mensajes, a través de diferentes dispositivos; en la mayoría de los casos esta información es amorfa, y también polimorfa. Bombardeo de imágenes, códigos, señales, lenguajes y signos que en ocasiones, por la rapidez con la que se dan, no otorgan el suficiente tiempo para que sean procesados conscientemente por la mente. 
Con esto queda claro que la realidad se muestra como un cuadro hecho con trazos difusos, amplios, inabordables y pasajeros, es decir, de difícil interpretación. Ante esto, la sociedad se encuentra sumergida en una especie de aturdimiento porque, a pesar del dominio sobre lo técnico, no encuentra el mismo éxito al tratar de ejercer control sobre el contenido.

Ante este panorama, el ser humano precisa de instrumentos de navegación y de herramientas de análisis que le permitan llevar a cabo una reflexión coherente y un análisis crítico de la gran cantidad de datos que se le presentan. Los niños, los jóvenes y los adultos requieren tener a su alcance instrumentos y estar capacitados para decodificar el cúmulo de información que reciben a diario.

La educación para la comunicación o educomunicación pretende desempeñar esa función: dotar al ciudadano, sea este chico o grande, de los utensilios necesarios para poner orden a ese enredo al que se enfrenta cada día, cada hora, cada minuto y cada segundo. La educomunicación aparece, entonces, como la herramienta infaltable para toda persona que aspire a considerarse alfabetizada audiovisualmente:

Es en relación con la alfabetización de la que venimos hablando, que aparece la educación en comunicación -también llamada educomunicación- como herramienta precisa para alcanzar dicha alfabetización audiovisual. El desarrollo de la educación en comunicación está vinculado a su integración en el currículo obligatorio (Zarandona, Basterretxea, Idoyaga \& Ramírez, 2008, p.122).

Como campo de estudio, interdisciplinar y transdisciplinar, la educomunicación abarca las dimensiones teórico-prácticas de dos disciplinas: la educación y la comunicación que, aunque históricamente han estado separadas, tienen mucho en común $\mathrm{y}$, de hecho, exigen ser abordadas así. En realidad, esta también es conocida en el contexto iberoamericano como educación en materia de comunicación, didáctica de los medios, comunicación educativa, alfabetización mediática o pedagogía de la comunicación; y media literacy o media education, en el contexto anglosajón (López \& Cinta, 2015).

De la educomunicación existen diferentes concepciones teóricas como, por ejemplo, la de Célestin Freinet, quien convencido de la necesidad de transformar el sistema educativo introdujo por primera vez en la historia un medio de comunicación en el ámbito escolar. La acción pedagógica puesta en práctica a través de la imprenta escolar permitió transformar los métodos memorísticos y mecánicos basados en la transmisión de contenidos por otros más acordes a la vida y la curiosidad de los educandos. Estos, motivados por la necesidad de conocer la realidad social para poder comunicarla a través del periódico escolar, aprendían por medio de la comunicación (Kaplún, 1998).

Ya a partir de los años setenta, varios hechos 
y autores entraron en escena para conformar lo que hoy se conoce como el cuerpo creciente de la educomunicación, entre ellos es destacable:

la influencia de la perspectiva crítica sobre las industrias culturales de la Escuela de Frankfurt, a través de autores como Adorno, Horkheimer y Marcuse; así mismo, los estudios de Comunicación para el Desarrollo en América Latina y las teorías de Marshall McLuhan sobre la comunicación. De igual manera la producción textual y las experiencias de educomunicadores en diferentes partes de la Tierra, entre los que se destacan: Paulo Freire, Mario Kaplún, Francisco Gutiérrez y Daniel Prieto Castillo, entre otros (Barbas, 2012, p.159).

Por aquella época, el Consejo Internacional de Cine y Televisión (CICT) de la Unesco definió la educación para la comunicación así:

Por educación en materia de comunicación cabe entender el estudio, la enseñanza y el aprendizaje de los medios modernos de comunicación y de expresión a los que se considera parte integrante de una esfera de conocimientos específica y autónoma en la teoría y en la práctica pedagógica, a diferencia de su utilización como medios auxiliares para la enseñanza y el aprendizaje en otras esferas del conocimiento como las matemáticas, la ciencia y la geografía (CICT, 1973, citado en Margalef, 2010, p.77).

Por su parte, la Unesco, finalizando la década de los años setenta, y en aras de complementar el concepto emitido por la CICT, puntualizó que la educomunicación encierra:

[...] todas las formas de estudiar, aprender y enseñar a todos los niveles, $[\ldots]$ y en toda circunstancia, la historia, la creación, la utilización y la evaluación de los medios de comunicación como artes prácticas y técnicas, así como el lugar que ocupan los medios de comunicación en la sociedad, su repercusión social, las consecuencias de la comunicación mediatizada, la participación, la modificación que producen en el modo de percibir, el papel del trabajo creador y el acceso a los medios de comunicación (Unesco, 1979, citada en Ministerio de Educación de España, 1994, p.17).

En tal sentido, en la Conferencia Nacional sobre Comunicación, Masterman (1993) señaló que este campo de estudios "[...] debe apuntar a aumentar el conocimiento por nuestros alumnos del funcionamiento de los medios de comunicación, del modo que tienen de producir un significado, de su forma de organización, de cómo construyen la realidad, y de la comprensión de esa 'realidad' por quienes la reciben” (p.193).

\section{Usos y apropiaciones de las TIC en el aula}

Un factor clave que se desprende del amplio desarrollo tecnológico gestado en las últimas décadas son las denominadas Tecnologías de la Información y la Comunicación (TIC), las cuales se han convertido en un tema de actualidad e interés dentro de la literatura. 
El término TIC, se ha venido empleando como parte de las tecnologías emergentes que en la actualidad han obligado a las sociedades a transformarse y tienen relación con el uso de los diversos medios informáticos que permiten el almacenamiento, procesamiento y difusión de los diferentes tipos de información. En síntesis, constituye un elemento que se ha adherido a la cultura en su totalidad: "la transformación que están produciendo en nuestros modos de hacer -aún las actividades más cotidianas-y la magnitud en que han potenciado nuestras capacidades, las han convertido en un elemento indispensable para individuos y sociedades" (Depetris et al., 2008).

El manejo de la información de medios como computadores, software y hardware se presenta tanto, que es una necesidad imperante del medio globalizado. Esto responde al nuevo esquema mundial que ha transformado el espacio en una sociedad del conocimiento. Señala sobre el tema Pérez (2005):

En el actual contexto social y económico las siglas TI, tecnologías de la información, y TIC, tecnologías de la información y comunicaciones, no son ajenas a ningún ámbito $\mathrm{y}$ forman parte de la vida cotidiana tanto de ciudadanos como de las empresas, donde indiscutiblemente se les reconoce como pilares básicos para el desempeño normal de la actividad humana (p.10).

En este orden de ideas, el desarrollo tecno- lógico ha posibilitado la ampliación y potenciación de tecnologías que permiten nuevas formas comunicativas y una mayor producción de la información, las cuales tienen incidencia global sobre las actividades humanas, y una de estas actividades se refiere a la producción y socialización del conocimiento.

Por su parte, Marqués (2001) describe que las TIC tienen múltiples funciones dependiendo del campo en el que se utilicen: económico, social, político y cultural. Dentro de las funciones relacionadas con la generación de conocimiento se encuentran:

- Posibilitar la comunicación, la transmisión de la información y la construcción de comunidades de aprendizaje autónomo.

- Potencializar los procesos a nivel de pensamiento, permitiendo que las personas construyan estructuras mentales y nuevas formas de pensar.

- Organizar, clasificar y analizar la información en términos de eficiencia para mejor manejo y mayor acceso por parte de la sociedad.

- Crear nuevos espacios y metodologías para la enseñanza y el aprendizaje, como el campo del e-learning, fortaleciendo procesos metacognitivos.

- Generar nuevos modelos de aprendizaje para las comunidades.

Uno de los referentes más importantes de la educomunicación es Paulo Freire, pedagogo brasilero, autor de la obra Pedagogía del oprimido, 
que enfatiza el poder liberador de la educación. Este autor establece una dialéctica entre el docente y el estudiante, a fin de potencializar los recursos creativos con los que cuentan ambos.

En las relaciones humanas del gran dominio, la distancia social existente no permite el diálogo. Este, por el contrario, se da en áreas abiertas, donde el hombre desarrolla su sentido de participación en la vida común. El diálogo implica la responsabilidad social y política del hombre (Freire, 2007, p.64).

Sin lugar a dudas, una sociedad que tiene el actual ritmo acelerado de información, la más alta competencia laboral de los últimos tiempos y el creciente agudizamiento tecnológico, debe estar en la capacidad de formar a su generación de relevo con las competencias necesarias en los ámbitos de conocimiento, pero también en el uso adecuado de las TIC.

Esta apreciación no es solo para los universitarios que escogieron la carrera de sus sueños, es también, y sobre todo, para los estudiantes de los colegios de cualquier año, que están sumergidos en una realidad interconectada y fugaz que les abre horizontes inexplorados, con todos los matices a su disposición. Pensar en la potencialidad que ofrecen las TIC, más allá del ocio o del proceso cultural, es posible cuando se establece una cobertura de educomunicación a través de la crítica y análisis de los usos y apropiaciones de estas herramientas.
Se trata de una educación que permita desarrollar la adaptación al cambio, así como un aprender que resalte la flexibilidad mental para operar con información abundante $\mathrm{y}$ diversa, pues no se puede olvidar que los niños viven inmersos en este mundo (Sánchez, 2001, p.42)

No se está hablando de incorporar un par de innovaciones tecnológicas al aula de clase, es de vital importancia identificar el contexto en el que se mueven los estudiantes. La virtualidad, las aplicaciones, los medios, la interacción globalizada, los nuevos discursos y demás elementos que conforman la cotidianidad de niños, adolescentes y jóvenes, deben vincularse en el proceso enseñanza-aprendizaje. Arriesgarse a establecer puentes sólidos entre la práctica pedagógica y la vida real que transcurre fuera de la institución es un llamado a la coherencia social.

Castells (2006) plantea que las tecnologías que utilizamos para interactuar en el mundo virtual tienen una relación dialéctica con la cultura, así mismo señala que muchos rasgos del paradigma informacional devienen también rasgos culturales, como son la interconexión, la porosidad y la flexibilidad (p.88).

La hibridación cultural da cuenta de la herencia de la globalización y de cómo este contacto al que se exponen los individuos irrumpe en las aulas de clase, sin distinción social permitiendo una afluencia de saberes que trasciende el espacio geográfico. Es cierto que las diferencias 
socioeconómicas enmarcan situaciones y posibilidades diferentes en cuanto al acceso de la información, pero es sabido que la proliferación de estas herramientas y medios tecnológicos han plagado países enteros y se han colado en lugares insospechados.

La implementación de estos medios, ya no es solo la elección de un par de docentes modernos que quieren volver su clase un show, esta práctica es un deber que se convierte en un privilegiado reto para algunos y un dolor de cabeza para otros.

En el campo de la educomunicación, comunicación cultural y para el cambio social, es necesario entender que "la transformación de los modos de leer... está dejando sin piso la obstinada identificación de la lectura con lo que atañe solamente al libro y no a la pluralidad y heterogeneidad de textos, relatos y escrituras (orales, visuales, musicales, audiovisuales, telemáticos) que hoy circulan” (Martín-Barbero,1997, p.12).

También es preciso conocer cómo se establecen líneas curriculares en las que penetre la nueva manera de leer el conocimiento, es decir, ya no es el libro el eje de este proceso, sino que ahora más bien se enfoca la dinámica desde una superficie enriquecida con el relato en tercera dimensión, multimedial y plurisensorial.

\section{Proceso metodológico}

A partir de los avances tecnológicos se ha generado una nueva visión de la sociedad en la que los adolescentes, como parte de ella, encuentran cada vez menos barreras de tiempo y espacio frente al uso de la Internet. Cada día son más numerosos los retos sobre la materia, y por tal razón la escuela se ve abocada a investigar para generar respuestas a las encrucijadas y problemáticas que plantean las interacciones entre los muchachos y los paisajes audiovisuales que se dibujan ante ellos a través de las TIC.

Siendo esta la pretensión del actual proyecto, en el cual se busca consolidar una estrategia educomunicativa para una aprehensión crítica de Facebook y YouTube en los jóvenes de noveno grado de instituciones de educación media de Cúcuta, Pamplona y Villa del Rosario. Caso: Colegio Departamental Integrado General Santander, se estableció llevar a cabo un tipo de investigación que permita entablar diálogo entre ideas y ejercicios sustentados en técnicas como la encuesta y el focus group.

El propósito central del estudio no se reduce solo a la descripción y al análisis del fenómeno social-educativo ya mencionado, sino al diseño de una propuesta formativa que permita la formación para consumir críticamente aquello que es transferido a través de las tecnologías y los medios de comunicación. Se trata de una propuesta centrada en la alfabetización audiovisual, pues los sujetos partícipes del estudio solo han llegado al dominio de lo técnico por iniciativa propia y de manera espontánea, pero requieren alcanzar mayores niveles formativos que les permita la decodificación de lenguajes, símbolos y 
mensajes que circulan en la web, para que logren adoptar una postura crítica frente al mismo.

En tal sentido, la investigación es de tipo descriptiva, y busca identificar los elementos que caracterizan determinado fenómeno objeto de análisis; es decir, se preocupa por la descripción detallada de la situación que es sometida a investigación a fin de ahondar en el problema y descubrir su naturaleza y estructura. Sobre este tipo de investigación sostiene Tamayo y Tamayo (2005) que se concentra en "la descripción, el registro, el análisis y la interpretación de la naturaleza actual, y la composición o procesos de los fenómenos" (p.46). En efecto, esta investigación se centra en la identificación de las habilidades críticas y acríticas de un grupo de estudiantes frente a contenidos de Facebook y YouTube en un marco donde se analizan factores como el equipamiento, la frecuencia, los usos y prácticas, las intencionalidades y las funciones, y la interpretación dada a estos contenidos web.

Por otro lado, atendiendo a que esta investigación se inscribe en un macroproyecto en donde se analiza el mismo fenómeno en escenarios diferentes, estudiantes de noveno grado en Cúcuta, Pamplona y Villa del Rosario, el proyecto corresponde a un estudio de carácter transversal que tiene como objetivo comparar un mismo fenómeno entre varios grupos en un único momento temporal.

\section{Población}

Este estudio se encuentra comprendido por jóvenes de noveno grado de las instituciones educativas de las ciudades de Cúcuta, Villa del Rosario y Pamplona (Colombia). En Cúcuta, el caso corresponde a la Institución Educativa $\mathrm{Pa}$ dre Luis Variara y Colegio San José de Cúcuta; en Pamplona, el caso está centrado en la institución educativa Colegio Cambridge School y en Villa del Rosario el caso analizado es el del Colegio Departamental Integrado General Santander.

En este documento se presentan los resultados y el análisis del Colegio Departamental Integrado General Santander-Sede Centro-de Villa del Rosario, cuya población está constituida por 560 estudiantes pertenecientes a la jornada de la tarde y que oscilan entre los grados séptimo y once (nivel de educación media).

\section{Muestra}

En la investigación sobre estrategias educomunicativas hacia una aprehensión crítica de los contenidos de Facebook y YouTube en los jóvenes de noveno grado de instituciones de educación media de Cúcuta, Pamplona y Villa del Rosario. Caso: Colegio Departamental Integrado General Santander, se utiliza el tipo de muestreo probabilístico, que es aquel en donde toda la población a intervenir tendrá la misma oportunidad de ser seleccionada a partir de los procesos que se realicen. A través de ello, se utilizó la aleatoriedad para garantizar la correcta muestra representativa de las unidades que componen el universo. 
A su vez, y con el fin de garantizar la validez de la investigación, se implementó un subtipo de muestra llamada muestreo probabilístico mixto, que presenta la combinación de dos tipos. Primero, muestreo probabilístico simple, en el que se asegura que toda la población esté incluida en el proceso para poder establecer de manera adecuada la selección al azar del número deseado de sujetos. Y segundo, muestreo probabilístico estratificado o proporcional, en el cual los individuos son agrupados en diferentes maneras, tales como edad y sexo, entre otras.

El primer tipo de muestro probabilístico es utilizado para garantizar los resultados precisos en la aplicación de la encuesta, y el segundo, para establecer la cantidad de estudiantes que integran el focus group.

\section{Diseño de la investigación}

Comprende las etapas o fases del estudio, es decir, el proceso que sigue el investigador para alcanzar los objetivos propuestos. En la literatura se hace referencia a diferentes etapas y fases que estructuran la investigación cualitativa. Para este estudio se consideran los aportes de Rodríguez, Gil y García (1996), quienes señalan que la investigación cualitativa sigue por lo general un conjunto de fases a saber: preparatoria, trabajo de campo, analítica e informativa.

\section{Interpretación general de los resultados}

El nivel de equipamiento tecnológico de los

\section{jóvenes de noveno grado}

Los resultados obtenidos a partir de la aplica- ción de las técnicas de investigación permitieron evidenciar el nivel de equipamiento tecnológico de los jóvenes que participaron en el estudio. Hablar de equipamiento significa ahondar en las oportunidades y capacidades de los sujetos para acceder a contenidos de Facebook y YouTube. Si las capacidades y oportunidades son mayores, el nivel de información al que es expuesto el sujeto también aumentará. Por tanto, las habilidades críticas se convierten en la opción requerida para que el estudiante logre asumir una posición adecuada sobre los contenidos que percibe en las redes sociales.

Estos jóvenes muestran que permanentemente tienen acceso a los contenidos de Facebook y YouTube, es decir, en su totalidad, la población participante del estudio tiene una cuenta de Facebook y visita YouTube para diferentes fines. El promedio de tiempo utilizado oscila entre una y dos horas diarias en la mayoría de los casos, y esto ocurre dos o tres veces por día. Ello significa que mínimo tres o cuatro horas destinan los jóvenes para tener contacto con los contenidos de estas dos redes. La disponibilidad de medios tecnológicos como tabletas o celulares de alta gama, que en la actualidad son más baratos que años atrás, han servido a esta generación para construir redes y comunicarse más abiertamente.

El tiempo y la frecuencia que destinan estos usuarios para acceder a Facebook y YouTube pone de manifiesto una nueva cultura caracterizada por la conectividad, la red y la virtualidad, donde los saberes formales e informales son so- 
cializados y aprehendidos a través de este tipo de medios. Como se observa en los resultados, a medida que disminuyen las limitaciones para la compra de equipos como portátiles, celulares, tabletas o ipads, aumentan las oportunidades para que los jóvenes se mantengan conectados a las redes sociales. Esto se demuestra al evidenciar que todos los participantes cuentan con algún tipo de tecnología que les facilita el acceso.

Para estas generaciones, es protagónico el rol que tienen la conectividad y la comunicación virtual en sus vidas diarias. Es evidente que existe una necesidad entre los jóvenes por hacer parte de esta nueva cultura donde recibir información y transmitir información se convierte en un espacio de socialización e interacción que les permite estar con los demás. De allí que la cantidad de tiempo empleado sea cada vez mayor entre los usuarios, y el acceso a las tecnologías resulte un asunto cuyas limitaciones se desvanecen con el desarrollo en este campo.

\section{Usos y prácticas de contenidos de Face-}

\section{book y YouTube entre estudiantes}

Los resultados evidencian que los jóvenes realizan un gran cúmulo de actividades en las redes Facebook y YouTube. En general, su actividad central es chatear con otras personas e informarse de manera general. Así, Facebook, y otras plataformas de este tipo, permanecen en constante transformación, para ofrecer a los usuarios diferentes elementos de atracción y asegurar su uso permanente. Detrás de ello se encuentra la ampliación del mercado y el incremento comer- cial, por lo que su uso no puede ser analizado de forma aislada a las pretensiones propias de los mass media.

En otros términos, el objetivo de las redes analizadas es alcanzar un grado de adaptación respecto de las necesidades y requerimientos de los usuarios para garantizar su permanencia en las mismas. Por ello, las funciones básicas de chat y publicación de estados, fotos, videos e imágenes es solo una parte de todas las posibilidades que tienen estas redes, ya que otras actividades pueden ser igualmente desarrolladas. En todo caso, la información que circula es cada vez mayor y la recepción de contenidos aumenta entre los usuarios, quienes toman diferentes percepciones sobre estos contenidos.

Valga aclarar que si bien el uso principal del Facebook es el chat, su acceso supone un acercamiento del usuario con los diferentes contenidos que son transmitidos por la red. A su vez, los participantes muestran una necesidad de acercamiento con los otros individuos por lo que la comunicación tiende al aumento. Por tanto, hoy más que nunca los jóvenes buscan comunicarse entre ellos mismos a través de códigos elaborados desde su particular cultura. Los significados, las actitudes, las percepciones y las posiciones son construidos por dichos actores de la comunicación, es decir, los jóvenes.

Por tanto, el problema no se encuentra en la comunicación y socialización de información elaborada por los jóvenes, sino en el tipo 
de contenidos elaborados y valorizados por los usuarios. Aquí resalta la tesis de propiciar una formación integral que les permita a los jóvenes asumir un papel dinámico, activo y serio frente a su realidad sociopolítica, económica y cultural con el fin de viabilizar transformaciones que a mediano y largo plazo faciliten la sostenibilidad del mundo en todos los niveles.

Al referirse a los riesgos que se desprenden de las redes sociales, los jóvenes que participaron en el estudio señalan que estos peligros dependen, en su mayoría, del uso que se tenga de estos medios. Poco se manifestaron sobre los riesgos que implica el contacto permanente con este tipo de sistemas donde se reduce la capacidad para centrarse en los problemas más urgentes de orden social. En otras palabras, la permanencia y el uso desmedido de estos medios pueden ocasionar que los sujetos desvíen su atención de los problemas colectivos y públicos relevantes reduciendo su participación. Y ello también configura un riesgo en este tipo de medios, pues los jóvenes destinan tiempo y esfuerzos para identificar contenidos que si bien para ellos son importantes, poco o nada contribuyen a una formación de la ciudadanía activa.

Por lo anterior, en el ámbito académico, la opinión de los sujetos en cuanto a la posibilidad de hacer uso de las redes Facebook y YouTube resulta dividida. Hay un número considerable de participantes dispuestos a utilizar estos medios en el campo educativo, pero también hay otros tantos que no se encuentran seguros de tal op- ción, por lo que están dejando a estas plataformas en el campo meramente recreativo, y ello se debe a que la utilidad asignada por años a este tipo de medios no ha incluido la de formación de conceptos y saberes formales. Los resultados se relacionan con el porcentaje del $84 \%$ de estudiantes que opina que la función de las redes como YouTube es de entretenimiento. Si bien los participantes reconocen la posibilidad de utilizar el Facebook y el YouTube en el campo formativo, así mismo poca claridad tienen sobre la forma como esto podría ser posible. De allí que en el uso específico de los contenidos de YouTube, las actividades centrales se reduzcan a ver videos y a visitar youtubers mientras que un número reducido de estudiantes lo hacen para fines educativos (tutoriales, compromisos escolares, entre otros).

\section{El grado de alfabetización audiovisual de los participantes}

Las capacidades críticas de los sujetos se reducen al análisis e interpretación de contenidos que muchas veces poco aportan a la construcción de un conocimiento formal. Por ello la mayoría de los jóvenes o bien toman como verdaderos o válidos los contenidos de estas redes, o ni se detienen en pensar en este tipo de aspectos. El porcentaje de jóvenes que afirma realizar actividades de consulta e investigación para validar los contenidos que perciben es menor al número de estudiantes que no ha pensado en la posibilidad de que los contenidos de las redes sean manipulados. Queda la duda sobre la forma como los sujetos confirman la veracidad de los contenidos 
y sobre qué tipo de contenidos ejercen el proceso de validación, pues de esto depende también las capacidades críticas que se tengan.

En efecto, una habilidad crítica es la de seleccionar contenidos que resulten de valor y utilidad para los usuarios en términos formativos: información que le permita a los actores reconocer su papel sociohistórico y las necesidades más urgentes de su contexto para participar de forma activa y dinámica en la construcción de mejores posibilidades. Al examinar detenidamente los resultados se observa que los estudiantes tienen contacto con una considerable cantidad de información que poca relevancia representa para su formación integral. Por ejemplo, basta con revisar en el desarrollo del focus group los ejemplos dados en cuanto a falsedad de la información: "Sí he sido víctima porque me crei la noticia de que Cristiano Ronaldo había donado 8 millones de euros a una fundación en Nepal y fueron puras mentiras" o "A veces uno lee en Facebook que se murió un actor, que un cantante se mató y cosas asi que resultan ser falsas". Estos contenidos son valorados por los estudiantes porque representan un interés para ellos, pero en sí el contenido no genera la posibilidad de adquirir una posición crítica del sujeto respecto de su contexto y los problemas próximos.

Básicamente, un porcentaje considerable de estudiantes concibe que el Facebook no tiene influencia en su comportamiento mientras otro número importante de sujetos señala que esto ocurre en algunas ocasiones. El solo hecho de que cada uno de los participantes tenga y acceda cotidianamente a una cuenta en la red varias veces al día, muestra una influencia en su comportamiento. Como se ha sostenido, la necesidad de pertenecer y sentirse integrado ha conducido a una transformación del comportamiento y de las actitudes de los jóvenes, que destinan un tiempo representativo en estas redes estudiadas.

Los hallazgos son coherentes con las opiniones de los estudiantes en el focus group, pues en su mayoría todos explican que hay una necesidad de pertenecer a las redes sociales. Para los participantes, la existencia y el uso colectivo de estas impulsan la necesidad de integración y participación: "Lo que pasa es que ahora es una necesidad, aunque antes no existian y todo era normal; pero como ya están, pues hacen falta” o "Antes había otras formas para comunicarse, pero en la actualidad existen las redes sociales y ya uno tiene que adaptarse a ellas y comunicarse con ellas". Del mismo modo la comparación de los hallazgos en las dos muestras que participaron en el focus group deja observar poca diferencia entre uno y otro resultado, excepto por la influencia de las redes: todo el segundo grupo que observó el video manifiesta que estos medios tienen influencia en la forma de pensar y actuar de los jóvenes, y parte del mal uso de Facebook y YouTube es permitir que las mismas redes demanden una cantidad de tiempo mal aprovechado.

Estos resultados se encuentran correlacionados con los hallazgos en cuanto a socialización 
y discusión de los contenidos entre los participantes y sus círculos sociales. Los contenidos analizados, sin que esto represente una habilidad crítica, y discutidos con otros pares infieren que: a) existe una necesidad de conocer sobre los contenidos para compartir con los otros (necesidad de integración), b) hay una dependencia de los jóvenes con otros actores, y c) la comunicación trasciende el espacio virtual para expandirse a otros escenarios reales.

\section{Estrategia educomunicativa para la apre-} hensión crítica de los contenidos de Facebook y You Tube

Una propuesta educomunicativa para la aprehensión crítica de contenidos de Facebook y YouTube exige un diseño a nivel pedagógico buscando fortalecer las habilidades críticas necesarias para la evaluación adecuada y oportuna de los contenidos a los que son expuestos los jóvenes. Una propuesta de este tipo está sustentada en los hallazgos preliminares encontrados con la aplicación de la encuesta y el focus group a estudiantes. En efecto, los resultados ponen de manifiesto que estos jóvenes:

- Tienen acceso de forma diaria a los contenidos de Facebook y YouTube a través de diferentes dispositivos, especialmente, celular y computadoras.

- Cuentan con habilidades de uso y manejo adecuados para aprovechar una propuesta basada en el enfoque educomunicativo.

- Dedican un tiempo considerable en las redes sociales expuestas para diferentes usos y actividades, y, por lo general, consideran estas herramientas como un medio que puede ser utilizado por la escuela.

- Prefieren los contenidos virtuales sobre los contenidos de otro tipo, v.g., libros o textos.

- Han ampliado habilidades técnicas para el uso adecuado de los medios tecnológicos y han aumentado su capacidad de comunicación a través de otro tipo de signos que la escuela debe reconocer y aprovechar.

- Reconocen que existen algunos riesgos con el uso de las redes Facebook y YouTube, pero que estos dependen de los usos así como de las acciones preventivas y correctivas que se apliquen.

- Pueden ser expuestos a una gran variedad de temas y contenidos que son de interés para los jóvenes y que la escuela debe identificarlos como medios para fines más complejos en términos formativos.

Con todo lo anterior, una propuesta o un modelo debe considerar varios elementos como son el proceso de enseñanza-aprendizaje, las intencionalidades o fines de la formación, los tiempos requeridos para este tipo de formación, las bases teórico-conceptuales que lo sustentan, los medios necesarios para llevar a cabo el proceso, el tipo de prácticas pedagógicas y docentes que se deben fortalecer. En este orden, se requiere hacer una aproximación previa a cada uno de estos elementos.

La concepción del proceso de enseñanzaaprendizaje en la propuesta

La educación se entiende como un concep- 
to complejo y dinámico en el cual se relacionan diversos factores asociados con los procesos de enseñanza-aprendizaje; en otras palabras, las interacciones que surgen entre docentes y estudiantes respecto del saber y la cultura que se busca transmitir. La relación entre docente, objeto de conocimiento (o saber) y estudiantes está dada por dos procesos esenciales, cada uno con características particulares, pero interdependientes: la enseñanza y el aprendizaje.

No hay lugar a duda que la enseñanza y el aprendizaje corresponden a procesos (Delacôte, 1998; Hervás, 2003; Martínez, 2004; Ibáñez \& Gómez, 2005), y ello se debe a que cada uno está integrado por etapas o fases interconectadas cuyo fin es la obtención de un resultado. En términos educativos, los resultados perseguidos por la enseñanza es el aprendizaje efectivo de los estudiantes y el resultado buscado por el aprendizaje es el cambio a nivel de comportamiento.

Esta perspectiva está sustentada por autores como Martínez (2004), para quien el aprendizaje corresponde al cambio que la persona tiene respecto de su conducta, es decir, las modificaciones que desarrolla internamente a fin de dar solución a un problema. Sin embargo, otros expertos en el tema consideran que el aprendizaje como cambio de comportamiento corresponde a una perspectiva de naturaleza conductista:

Es bien cierto que, durante muchos años, los conductistas definían el aprendizaje como un cambio en la conducta. De esta forma que- daban excluidos los efectos del aprendizaje latente [...] que se produce en ausencia de conductas observables. Sin embargo, la necesidad de que el aprendizaje se manifieste en una conducta observable solo se sigue de las asociaciones E-R o R-E, que implican una respuesta por parte del sujeto (Pozo, 2006, p.28).

Como respuesta al paradigma conductista, otros enfoques con posterioridad han abordado el proceso de aprendizaje como objeto de estudio y han replanteado dicho enfoque. Dentro de estos se encuentran aquellas teorías de corriente cognitiva como el constructivismo y el aprendizaje significativo, los cuales exploran el aprendizaje de forma alternativa al conductismo.

Moreira, Caballero y Rodríguez (1997) definen el aprendizaje como "el proceso a través del cual una nueva información (un nuevo conocimiento) se relaciona de manera no arbitraria y sustantiva (no-literal) con la estructura cognitiva de la persona que aprende" (p.20). Ahora bien, la enseñanza correspondería a las acciones diseñadas y planificadas por el docente para ejercer el acto pedagógico que viabiliza el aprendizaje del estudiante, y frente a él diversos elementos y factores confluyen como son las capacidades y habilidades del docente, los recursos y medios implementados, los tiempos empleados para la enseñanza, los tipos de contenidos, las formas de evaluación que emplea, entre otros. La manera como el docente organiza y utiliza cada uno de estos elementos dan lugar a las denominadas prácticas pedagógicas. 
Las prácticas pedagógicas están referidas a todos los procedimientos, estrategias y acciones que estructuran la interacción, la comunicación, las relaciones y las posiciones de los principales sujetos que intervienen en el acto educativo, es decir, los docentes y los estudiantes (Díaz, 1993). Ahora bien, cada uno de estos procedimientos, estrategias y acciones implementadas en los procesos de enseñanza por parte de los docentes se encuentra relacionado de forma directa con las perspectivas, concepciones y representaciones que soportan la actividad de los docentes, y por ello Ordóñez (2004) indica que la pedagogía debe ser entendida "como una disciplina que se mueve entre la teoría y la práctica, y que debe valerse de la primera para crear formas de mejorar la efectividad de la segunda en el aprendizaje de las personas, por medio de la experiencia y la investigación, de cualquier naturaleza que esta sea" (p.7).

En otras palabras, las prácticas pedagógicas tienen como antesala las concepciones filosóficas y epistemológicas que han construido los docentes sobre lo que significa la educación. De allí que no sean universales ni homogéneas, y, por el contrario, correspondan a una gran diversidad de acciones que se desarrollan con la finalidad de impactar positivamente en el aprendizaje de los sujetos y que dependen de la naturaleza de los conocimientos que se buscan transmitir. En este mismo sentido concibe Latorre (2004) las prácticas pedagógicas, para quien mantienen una doble perspectiva: acción realizada y sentido atribuido a la acción, es decir, conjunto de acciones con intencionalidad o propósito que se orga- nizan y estructuran conforme a las exigencias y características del medio.

Con todo lo anterior, la propuesta pedagógica de capacitación o formación para estudiantes de educación media sobre uso crítico de los contenidos de Facebook y YouTube se encuentra sustentada en que su finalidad es el aprendizaje del sujeto de formación. En otras palabras, el proceso de enseñanza-aprendizaje está sustentado en las bases de las corrientes cognitivas y el enfoque crítico de la educación donde lo importante es la identificación de la información, el tratamiento dado a esta información y la capacidad para tomar una postura político-social clara respecto de los contenidos de las redes sociales.

Las prácticas pedagógicas de los docentes bajo esta propuesta no tienen el objetivo de enseñar contenidos. Por el contrario, a partir de contenidos o temas específicos busca ahondar con los estudiantes en diferentes tipos de capacidades y habilidades para desarrollarlas. Estas prácticas se deben caracterizar por la flexibilidad, el uso permanente del diálogo y la comunicación, el diseño de acciones a partir de propuestas colectivas y la definición clara de intencionalidades respecto de las acciones.

\section{Intencionalidades o fines de la propuesta} educomunicativa

Los fines o intencionalidades de la propuesta de formación son los siguientes: a) desarrollar habilidades críticas para el manejo acertado de contenidos de Facebook y YouTube, b) fortalecer la participación y las prácticas ciudadanas de 
los estudiantes en función de un reconocimiento de lo público y una mayor aceptación y respeto de las diferencias, c) desarrollar habilidades cognitivas y metacognitivas que les permita a los estudiantes el tratamiento adecuado de la información, d) desarrollar las habilidades de uso y manejo de las TIC entre los estudiantes, y e) colaborar en la construcción de un sujeto sociopolítico con capacidad para identificar los problemas de su contexto y proponer alternativas de solución.

\section{El papel de los contenidos}

Como se indicara con anterioridad, en esta propuesta se escoge un conjunto de contenidos o temas que servirán como medios para lograr habilidades críticas en pos de la evaluación objetiva de los contenidos de redes sociales. Por lo tanto, el docente no se centrará en preguntar o explorar la capacidad de memoria del estudiante sobre los contenidos a los que son expuestos durante la evaluación, sino que buscará las acciones o estrategias que permitan identificar el grado de avance respecto de habilidades críticas como: identificación y selección de información, análisis de información, codificación y decodificación de información, comparación de contenidos, capacidad de abstracción del estudiante a otros escenarios, capacidad de resolver problemas en diferentes niveles, ampliar la capacidad de comunicación y diálogo con sus pares, habilidad para validar la información a la que es expuesto, entre otras.

Atendiendo a los hallazgos, los contenidos deben encontrarse en las redes Facebook y You-
Tube, ya sea en formato de imagen, video, audio u otro. Estos serán identificados por los docentes para el diseño de estrategias pedagógicas. Así mismo, el docente debe tener como criterio para la selección de contenidos aquellos que representen un problema de naturaleza sociopolítica o económica de tal manera que los sujetos en formación puedan transformar su escala de valoración en cuanto a la pertinencia de los contenidos.

\section{Los medios requeridos para el proceso for-} mativo

Conforme a los resultados obtenidos en la investigación, los dispositivos tecnológicos que se utilizan para el desarrollo de la propuesta son celulares android o computadoras. Por lo anterior, el docente se integrará a estos espacios para proponer la exploración sistemática y adecuada de los contenidos, que pueden ser discutidos en las mismas plataformas o redes, y ampliados en el aula de clase a través de procesos de lectura y escritura formal, $\mathrm{u}$ otras actividades significativas de aprendizaje experiencial.

De igual manera, elaborará material audiovisual y campañas mediáticas de corte on line que puedan ser percibidas por los educandos a través de sus ordenadores; la reflexión crítica de estos contenidos va a generar al mismo tiempo procesos de alfabetización mediática.

\section{El papel de los sujetos vinculados al proce-} so de enseñanza-aprendizaje

El estudiante tiene un papel protagónico en esta propuesta pedagógica. Los intereses de naturaleza sociopolítica de esta población serán 
identificados con el propósito de dar sentido y significado a la actividad pedagógica. Predomina la comunicación desde los significados y la experiencia de los sujetos, y la información producida por los estudiantes será objeto de análisis para el docente. El estudiante se convierte en el principal impulsor de su proceso de aprendizaje, y ello conduce a la necesidad de fortalecer habilidades metacognitivas necesarias para fomentar la autonomía e independencia del mismo sujeto.

Por otro lado, el docente pasa a ser un media-

Gráfico 1. Estrategia educomunicativa para la aprehensión crítica de contenidos

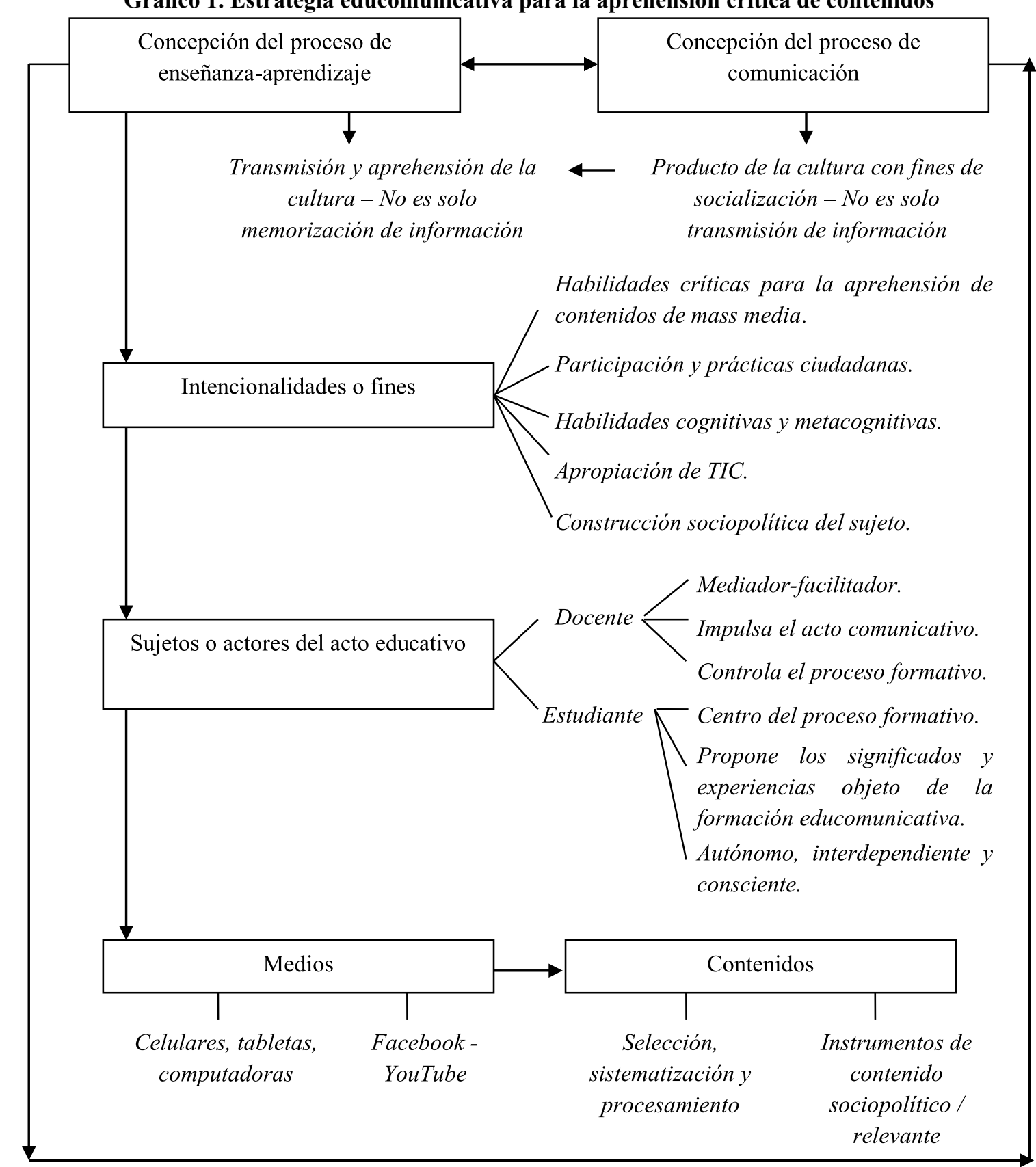

Fuente: Elaboración propia 
dor del proceso formativo; sin imponerse, guía a los estudiantes con preguntas concretas y claras que les permita identificar la finalidad de la actividad y el objetivo del proceso formativo.

\section{Conclusiones}

Los elementos tecnológicos corresponden a medios didácticos que pueden ser utilizados en el contexto de los procesos de enseñanzaaprendizaje. La relación entre estas dos esferas se halla en el campo de la comunicación y la mediación ejercida por los docentes, y puede convertirse en un instrumento de gran potencial en las escuelas. Y en este proceso comunicativo bidireccional y enriquecedor, los elementos tecnológicos se muestran como parte de la nueva sociedad de la información, una organización social caracterizada por el manejo de la información a través de las tecnologías que permiten la construcción de escenarios complejos y cooperativos de aprendizaje. En este orden de ideas, los docentes deben ser formados en el uso pedagógico de las tecnologías para que descubran y evidencien sus potencialidades dentro del aula, es decir, cómo pueden ser útiles para fortalecer la virtualidad, la interactividad, el aprendizaje colectivo, el aprendizaje basado en elementos socioculturales, la construcción de conceptos y el aprendizaje complejo.

La educomunicación corresponde entonces a un campo de estudio pertinente y relevante para el contexto educativo actual. El auge de las TIC y el uso cada vez mayor de las redes sociales ponen de manifiesto la necesidad de formar a los jóvenes en competencias y habilidades que les permita la aprehensión crítica de los contenidos virtuales transmitidos en redes como Facebook y YouTube. En la investigación realizada se logró determinar que los estudiantes de noveno grado de la institución educativa Colegio Departamental Integrado General Santander, en su totalidad, hacen uso de estas redes de forma diaria y a través de diferentes dispositivos.

Lo anterior representa una oportunidad para la escuela, la cual puede aprovechar la conectividad en la que se encuentran inmersos los sujetos de formación para fortalecer los procesos de enseñanza-aprendizaje e impulsar habilidades en busca de mejorar su crecimiento y desarrollo personal. Pero esto solo es posible en la medida que los sujetos cuenten con capacidades para seleccionar, analizar, comprender e interpretar el amplio bagaje de información que circula en las redes, facilitándoles la construcción de una posición crítica.

La posición crítica del sujeto corresponde a una capacidad del individuo para comprender e interpretar su contexto, identificar los elementos de su entorno y extraer de este proceso el papel que le corresponde desempeñar desde el campo sociopolítico y ciudadano. En efecto, no basta que el sujeto de formación logre manejar y sistematizar los contenidos virtuales, pues debe determinar la información relevante que se moviliza en las redes como Facebook o YouTube, y con ello realizar abstracciones a su propio contexto en particular. 
Los resultados producto de la aplicación de las diversas técnicas demostraron que el nivel de equipamiento de los estudiantes facilita la adopción de una estrategia educomunicativa por parte de la escuela, pues los estudiantes cuentan con las capacidades para el acceso y el manejo de las TIC conforme el uso de las redes como Facebook y YouTube. Esto infiere también que los jóvenes tienen habilidades, conocimientos y saberes en el manejo de los medios que pueden ser aprovechados por los docentes y la escuela. Corresponden a presaberes capaces de impulsar un aprendizaje significativo entre los estudiantes, ya que en la medida en que dichas estructuras se encuentran interiorizadas (ideas de anclaje), los nuevos conceptos tienen mayores probabilidades de ser integrados a nivel cognitivo.

Los usos y prácticas de los jóvenes frente al Facebook y YouTube denotan dos aspectos sobresalientes: primero, el contexto de los sujetos está dado en parte con la conectividad y la virtualidad. Allí buscan comunicarse con los demás satisfaciendo la necesidad de estar con los otros de una manera u otra, ya sea interactuando o como usuarios receptivos de información. Por ello las principales actividades realizadas son chatear, actualizar información o revisar la actualización de los estados de las demás personas; segundo, los contenidos a los que son expuestos los jóvenes poco o nada tienen que ver con ejes o líneas que puedan ser asociadas con la formación sociopolítica y ciudadana. Esto se traduce en un gran cúmulo de información que distrae su atención y que desde la estrategia educomunicativa busca ser afrontado.
En otras palabras, la estrategia educomunicativa busca centrar a los jóvenes en los problemas reales de su contexto y su realidad, ya que las redes han servido en la mayoría de los casos para ofrecer espacios de entretenimiento. Se debe volver a un conjunto de problemas que requieren ser discutidos: la violencia generalizada, la xenofobia, el manejo adecuado de las emociones, el sentido de lo público, la participación democrática, entre otros. La finalidad es que los estudiantes reconozcan las oportunidades de los mass media, pero así mismo que observen las intencionalidades que impone el sistema en su búsqueda de crecimiento del mercado, a fin de desarrollar posiciones críticas que van a facilitarles la selección y el tratamiento de la información.

Y es que esta distracción va más allá de las redes. Muchos contenidos con poca relevancia se convierten en el tema de discusión en espacios reales, privando a los jóvenes de adentrarse $\mathrm{y}$ ahondar en los problemas colectivos y sociales que viven de forma diaria en sus barrios, escuelas o calles. Si bien las redes han mostrado casos en los que su uso puede fortalecer la movilización social y la adopción de posturas colectivas de contenido sociopolítico, también es cierto que este tipo de situaciones no se presentan en el caso particular estudiado.

Por lo anterior, se requiere de una estrategia educomunicativa que le facilite a los jóvenes navegar entre el amplio cúmulo de información que se moviliza en las redes. El reto en particular 
es propiciar prácticas y saberes que en el campo práctico les ayude a seleccionar y omitir información a fin de que su formación se haga de manera sistematizada sobre temas que viabilicen la construcción sociopolítica y ciudadana. Por ello en la propuesta el rol principal del docente atiende la selección de los contenidos y su mediación frente a los jóvenes, pues son ellos quienes fortalecerán su autonomía y su capacidad de decisión en su papel protagónico como estudiantes.

\section{Referencias}

Aparici, R. (Coord.). (2011). Educomunicación: más allá del 2.0. Barcelona: Gedisa.

Ballesta, J. (Coord.) (2002). Medios de comunicación para una sociedad global. Murcia: Universidad de Murcia.

Barbas, Á. (2012). Educomunicación: desarrollo, enfoques y desafíos en un mundo interconectado. Foro de Educación, 14, 157-175.

Blázquez, F. (Coord.) (2001). Sociedad de la información y la comunicación. Mérida: Junta de Extremadura/Consejería de Educación, Ciencia y Tecnología.

Cabero, J. (1996). Nuevas tecnologías, comunicación y educación. Revista Electrónica de Tecnología Educativa, 1, 14-25.

Cárdenas, W. (2008). ¿Qué haría usted si no pudiera ver televisión? Una pregunta a niños y jóvenes para múltiples reflexiones con padres y maestros. Pamplona: Lito Flórez.

Castells, M. (1999). La era de la información. La sociedad red. Madrid: Siglo XXI Editores.
Castells, M. (2002). Tecnología de la información y la comunicación y desarrollo global. Revista de Economía Mundial, 7, 91107.

Castells, M. (2006). La sociedad de la información y sus contradicciones. Madrid: Siglo XXI Editores.

Delacôte, G. (1998). Enseñar y aprender con nuevos métodos. Barcelona: Gedisa.

Depetris, B., Feierherd, G., De Giusti, A., Sanz, C., González, A. \& Pousa, A. (2008). TIC en Educación. Trabajo presentado en el $\mathrm{X}$ Seminario de Investigadores en Ciencias de la Computación, Red de Universidades con Carreras en Informática, Argentina.

Díaz, M. (1993). La docencia como práctica pedagógica. Cali: Universidad del Valle.

Druetta, D. (2002). Sociedad de la información y el conocimiento. Entre el optimismo y la desesperanza. Revista Mexicana de Ciencias Políticas y Sociales, 45(185), 13-33.

Freire, P. (2007). La educación como práctica de la libertad. Montevideo: Siglo XXI Editores.

García, J. \& Álvarez, G. (2001). Reconfiguración como sujetos de comunicación: implicaciones para los ambientes virtuales con fines educativos. Revista de Universidad y Sociedad del Conocimiento, 5(2), 5-16.

Hervás, R. (2003). Estilos de enseñanza y aprendizaje en escenarios educativos. Granada: Grupo Editorial Universitario.

Ibáñez, V. \& Gómez, I. (2005). La interacción y la regulación de los procesos de ense- 
ñanza-aprendizaje en la clase de ciencias: análisis de una experiencia. Enseñanza de las Ciencias, 23(1), 97-110.

Kaplún, M. (1998). Una pedagogía de la comunicación. Madrid: Editorial La Torre.

Latorre, M. (2004). Aportes para el análisis de las racionalidades presentes en las prácticas pedagógicas. Estudios Pedagógicos, (30), 75-91.

López, L. \& Cinta, M. (2015). La docencia sobre alfabetización mediática en las Facultades de Educación y Comunicación. Comunicar, 44(22), 187-195.

Margalef, J. (2010). Retos y perspectivas de la educación mediática en España. Madrid: Ministerio de Educación, Cultura y Deporte.

Marqués, P. (2001). Las TIC y sus aportes a la sociedad. Barcelona: UAB.

Martín-Barbero, J. (1984). De la comunicación a la cultura: perder el "objeto" para ganar el proceso. Signo y Pensamiento, 3(5), 21-29.

Martín-Barbero, J. (1992). Pensar la sociedad desde la comunicación. Un lugar estratégico para el debate a la modernidad. Diálogos de la Comunicación, 32, 1-8.

Martín-Barbero, J. (1997). Heredando el futuro. Pensar la educación desde la comunicación. Nómadas, 5, 1-13.

Martínez, E. (2004). El proceso de enseñanza y aprendizaje. Madrid: ICE de la Universidad Complutense.

Masterman, L. (1993). La enseñanza de los medios de comunicación. Madrid: De la Torre.
Ministerio de Educación de España (1994). Guía para el uso de los medios de comunicación. Madrid: Autor.

Ministerio de Educación Nacional (2013). Competencias TIC para el desarrollo profesional docente. Bogotá: MEN/Corporación Colombia Digital.

Moreira, M., Caballero, M. \& Rodríguez, M. (1997). Actas del Encuentro Internacional sobre el Aprendizaje Significativo. Madrid: Burgos.

Murciano, M. (2006). Las políticas de comunicación ante los retos del nuevo milenio: pluralismo, diversidad cultural, desarrollo económico y tecnológico y bienestar social. Zer, 20, 371-398.

Ordóñez, C. (2004). Pensar pedagógicamente desde el constructivismo. De las concepciones a las prácticas pedagógicas. Revista de Estudios Sociales, 19, 7-12.

Pérez, D. (2005). Contribución de las tecnologías de la información a la generación de valor en las organizaciones: un modelo de análisis y valoración desde la gestión del conocimiento, la productividad y la excelencia de la gestión. Tesis doctoral no publicada, Universidad de Cantabria, España.

Pozo, J. I. (2006). Teorías cognitivas del aprendizaje. ( $9^{\mathrm{a}}$ ed.). Madrid: Ediciones Morata.

Rodríguez, E. (2009). El rol de las universidades en la sociedad del conocimiento y en la era de la globalización: Evidencia desde Chile. Interciencia, 34(11), 822-829. 
Rodríguez, G., Gil, J. \& García, E. (1996). Metodología de la investigación cualitativa. España: Ediciones Aljibe.

Sánchez, J. (2001). Aprendizaje visible, tecnología invisible. Santiago de Chile: Ediciones Dolmen.

Tamayo y Tamayo, M. (2005). El proceso de la investigación cientifica: incluye evaluación y administración de proyectos de investigación. México: Limusa.
Thompson, J. B. (1998). Los media y la modernidad. Una teoría de los medios de comunicación. Buenos Aires: Paidós Ibérica.

Tubella, I. \& Requena, J. (2005). Sociedad del conocimiento. Barcelona: Editorial UOC. Zarandona, E., Basterretxea, J., Idoyaga, P. \& Ramírez, T. (2008). La alfabetización audiovisual entre adolescentes vascos: implicaciones para las prácticas educativas y propuesta de intervención. Comunicación y Sociedad, 9, 119-143. 